\title{
Partial least squares based gene expression analysis in renal failure
}

\author{
Shuang Ding, Yinhai $\mathrm{Xu}$, Tingting Hao and Ping $\mathrm{Ma}^{*}$
}

\begin{abstract}
Background: Preventive and therapeutic options for renal failure are still limited. Gene expression profile analysis is powerful in the identification of biological differences between end stage renal failure patients and healthy controls. Previous studies mainly used variance/regression analysis without considering various biological, environmental factors. The purpose of this study is to investigate the gene expression difference between end stage renal failure patients and healthy controls with partial least squares (PLS) based analysis.
\end{abstract}

Methods: With gene expression data from the Gene Expression Omnibus database, we performed PLS analysis to identify differentially expressed genes. Enrichment and network analyses were also carried out to capture the molecular signatures of renal failure.

Results: We acquired 573 differentially expressed genes. Pathway and Gene Ontology items enrichment analysis revealed over-representation of dysregulated genes in various biological processes. Network analysis identified seven hub genes with degrees higher than 10, including CAND1, CDK2, TP53, SMURF1, YWHAE, SRSF1, and RELA. Proteins encoded by CDK2, TP53, and RELA have been associated with the progression of renal failure in previous studies.

Conclusions: Our findings shed light on expression character of renal failure patients with the hope to offer potential targets for future therapeutic studies.

Virtual Slides: The virtual slide(s) for this article can be found here: http://www.diagnosticpathology.diagnomx.eu/vs/ 1450799302127207

Keywords: Renal failure, Partial least squares, Gene expression, Network

\section{Background}

Renal failure refers to the medical condition that kidneys fail to adequately filter waste products from blood. It is usually not reversible and patients with end stage renal failure have to be treated with long term dialysis or organ transplant [1,2]. Preventive and therapeutic options for this disease are still limited [3]. Capture the gene expression signature of end stage renal failure patients may enhance the development of novel therapeutic strategies.

High throughput microarray analysis is powerful to characterize the underlying pathogenesis of various diseases. Several studies have investigated the gene expression difference between renal failure patients and controls using this strategy [4-6]. These studies generally carried out variance or regression analysis to

\footnotetext{
* Correspondence: pingm62@aliyun.com

Department of medical laboratory, The affiliated hospital of Xuzhou Medical College, No.99 Huaihaixi Road, Xuzhou 221000, China
}

detect dysregulated genes. This statistical procedure ignored unaccounted array specific factors, including various biological, environmental factors. Previous studies $[7,8]$ have suggested that partial least squares (PLS) based expression profile analysis is efficient in dealing with large amount of genes and fairly small samples. Compared with variance and regression analysis, PLS based analysis is more sensitive while maintaining reasonable high specificity, small false discovery rate and false non-discovery rate. Previous study using PLS analysis on other complex disease such as breast cancer has proved its feasibility [9]. Therefore, capturing the gene expression signature in renal failure patients by using PLS based analysis may provide new understanding of the pathogenesis and offer potential therapeutic targets.

In the current study, to investigate the gene expression difference between end stage renal failure patients and healthy controls, we performed PLS-based analysis by 
using gene expression data from the gene expression omnibus (GEO) database. Pathways or Gene Ontology items significantly over-represented with dysregulated genes were also acquired by using enrichment analysis. In addition, we constructed a protein-protein interaction (PPI) network with the proteins encoded by dysregulated genes to identify hub genes that may be related with disease progression.

\section{Methods}

\section{Microarray data}

The whole data set of gene expression profile GSE37171 from the GEO database was downloaded. This series represents transcription profile of 63 end-stage renal failure patients and 20 healthy controls. All samples were taken from peripheral blood. The dataset was based on the GPL570 platform ([HG-U133_Plus_2] Affymetrix Human Genome U133 Plus 2.0 Array). This study is approved by the institutional review board of the affiliated hospital of Xuzhou medical college (NO. 131081).

\section{Identification of differentially expressed genes}

Normalization of raw intensity values was performed by using Robust Multi-array Analysis (RMA) [10]. The resulting $\log 2$-transformed expression value of each probe was used in subsequent analysis. A multivariate linear model was used to describe the relationship between gene expression values and the disease status. For each sample, the model is expressed as:

$$
y=\sum_{i=1}^{p} \alpha_{i} x_{i}+b
$$

where $y$ is the binary variable of disease status, 0 coded as control and 1 coded as renal failure; $p$ is the total number of genes in the array. PLS analysis was then carried out to estimate the effects of each gene. The main purpose of PLS regression was to build orthogonal components (called 'latent variables' here). It is:

$$
\operatorname{COV}\left(\mathrm{t}_{\mathrm{k}} \cdot \mathrm{u}_{\mathrm{k}} \rightarrow \max \right)
$$

$$
\text { Subject to }\left\|t_{k}\right\|=1 \text { and }\left\|u_{k}\right\|=1
$$

where $\boldsymbol{t}_{k}$ is the $k$ th latent variable decomposes from all individuals' genes expression data $X$ (the matrix of $n \times p, n$ refers to the number of individuals and $p$ refers to the number of genes), $\boldsymbol{u}_{\boldsymbol{k}}$ is the $\boldsymbol{k}$ th latent variable decomposes from the phenotype data Y $(n \times 1)$ [11]. The non-linear iterative partial least squares (NIPALS) algorithm [12] was used to calculate the PLS latent variables derived from the expression profile on the target trait, as follows:

1) Randomly initialize $u_{0}=Y$

2) $w=X^{T} u_{0}, w=w /\|w\|$

3) $t=X w$
4) $c=Y^{T} t, c=c /\|c\|$

5) $u=Y c$

6) if $u-u_{0}<10 \mathrm{E}-8$, go to step 7), else $u_{0}=u$, repeat step 2)-5)

7) $X=X-t t^{\mathrm{T}} X, Y=Y-t t^{\mathrm{T}} Y$

Then go back to 2) to calculate the next latent variable.

To evaluate the importance of the expressed genes on disease, the statistics of variable importance on the projection (VIP) [13] was calculated as:

$$
V I P_{j}=\sqrt{\frac{p \sum_{k=1}^{h} \operatorname{Cor}^{2}\left(Y, t_{k}\right) w_{k j}^{2}}{\sum_{k=1}^{h} \operatorname{Cor}^{2}\left(Y, t_{k}\right)}}
$$

where, the Cor operator is the Pearson correlation coefficient, and for each $\boldsymbol{w}_{\boldsymbol{k}}$, it should be normalized by dividing $\left\|w_{k}\right\|$, and $h$ is the number of latent variables used in the model.

To avoid the model over fitting, the best number of latent variables ( $h$ above) was determined by the prediction accuracy based on three folds cross validation. The VIP for each gene was then calculated with the $h$ latent variables to obtain genes associated with renal failure. In addition, the false discovered rate (FDR) procedures were used to control the expected proportion of incorrectly rejected null hypotheses. The permutation procedure $(N=10000$ times) was used to obtain the empirical distribution of PLS-based VIP in each replicate. The FDR for each gene was then calculated as:

$$
F D R_{i}=\left(\sum_{j}^{10000} \sum_{i}^{p} \operatorname{Bool}\left(V I P_{i, j}>V I P_{i}\right)\right) /(10000 p)
$$

where Bool represents the logical value of expression: "True" codes as 1 and "False" codes as 0 . Significant genes were selected with a threshold of FDR $<0.01$.

\section{Enrichment analysis}

Annotation of all probes was carried out by using the simple omnibus format in text (SOFT) files. To capture biologically relevant character of differentially expressed genes, enrichment analysis was implemented. All genes were firstly mapped to the Kyoto Encyclopedia of Genes and Genomes (KEGG) pathways (http://www.genome.jp/ kegg/) [14] and Gene Ontology database [15]. Biological processes significantly overrepresented with differentially expressed genes were identified by using the hyper geometric distribution test.

\section{Network analysis}

PPI is important for all biological processes since most protein function through its interaction with other proteins [16]. Among the proteins encoded by differentially 
expressed genes, those with more interactions with other proteins may play more important roles in the progression of renal failure. To visualize the interaction among these proteins and identify key molecules, a network was constructed by using the software Cytoscape (V 2.8.3, http://www.cytoscape.org/) [17]. The database (http:// ftp.ncbi.nlm.nih.gov/gene/GeneRIF/) of NCBI was used to get the interaction information of all proteins. For each protein, the number of links (interactions) was defined as its degree. Proteins with degrees over 10 were selected as hub molecules in this study.

\section{Results}

According to the prediction accuracy based on cross validation, six latent variables were used in the detection of differentially expressed genes (Figure 1). The results revealed that 573 genes were differentially expressed between end-stage renal failure patients and healthy controls, including 141 downregulated genes in patients and 432 upregulated ones. For all genes in the array, 6084 genes were mapped to various pathways, including 203 differentially expressed genes. The pathways enriched with differentially expressed genes are listed in Table 1. These pathways are involved in several systems, including nervous system, digestive system, and endocrine system. In addition, three cancer pathways, transcriptional misregulation in cancers (hsa05202), chronic myeloid leukemia (hsa05220) and small cell lung cancer (hsa05222) were also enriched with differentially expressed genes. A total of 16517 genes in the array were annotated based on the GO database, including 518 differentially expressed genes.
Table 2 represents the five GO items enriched with selected genes. Protein binding (GO: 0005515) was the most significant GO item with over represented selected genes. In consistent with the pathway analysis, a transcription related GO item: transcription, DNA-dependent (GO: 0006351) was also identified to be overrepresented with dysregulated genes.

Figure 2 illustrates the interaction network of proteins encoded by differentially expressed genes. Seven proteins, CAND1, CDK2, TP53, SMURF1, YWHAE, SRSF1, and RELA were identified to be hub molecules, with degrees of $31,29,22,19,15,12$, and 10 respectively.

\section{Discussion}

Renal failure is a complex medical condition which may result from kidney injury or chronic diseases $[18,19]$. Microarray is a powerful technology for investigating the gene expression difference between end-stage renal failure patients and healthy controls. However, it is challenging to develop a suitable statistical model to deal with the small sample number and fairly large amount of genes. Previous studies on renal failure mainly used variance or regression analysis, without considering unaccounted array specific factors. Here we used PLS based analysis to identify dysregulated genes in end-stage renal failure patients.

Pathway enrichment analysis revealed that overrepresentation of dysregulated genes in various systems. Dysfunction of various systems may be complications of renal failure since kidneys are essential in the maintenance of homeostatic status. In addition, we also detected cancer-related pathways and GO items to be enriched

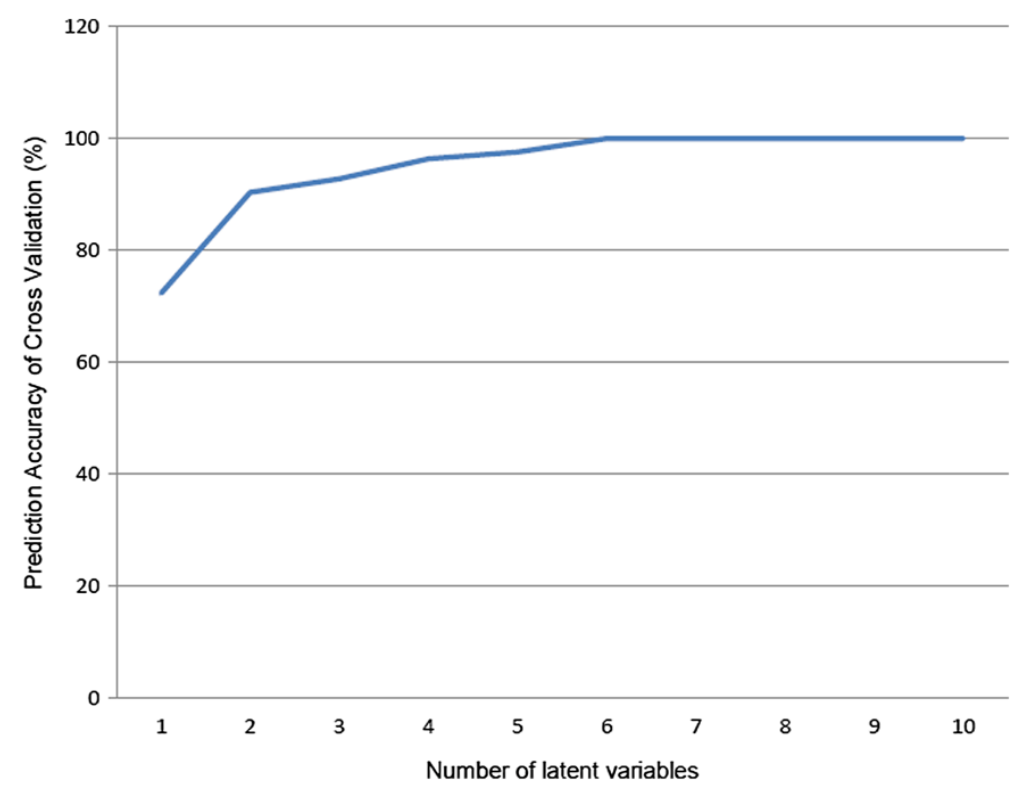

Figure 1 The distribution prediction accuracy as the number of latent variable number increases. The prediction accuracy achieves $100 \%$ when the latent variable number is six. 
Table 1 Pathways enriched with differentially expressed gene

\begin{tabular}{llll}
\hline KEGG id & Pathway description & Pathway class & P-value \\
\hline hsa04722 & Neurotrophin signaling pathway & Nervous system & $2.09 \mathrm{E}-03$ \\
hsa05202 & Transcriptional misregulation in cancers & Cancers & $5.20 \mathrm{E}-03$ \\
hsa04120 & Ubiquitin mediated proteolysis & Folding, sorting and degradation & $5.22 \mathrm{E}-03$ \\
hsa05220 & Chronic myeloid leukemia & Cancers & $1.05 \mathrm{E}-02$ \\
hsa05222 & Small cell lung cancer & Cancers & $2.28 \mathrm{E}-02$ \\
hsa05010 & Alzheimer's disease & Neurodegenerative diseases \\
hsa04970 & Salivary secretion & Digestive system & $2.43 \mathrm{E}-02$ \\
hsa04130 & SNARE interactions in vesicular transport & Folding, sorting and degradation \\
hsa04912 & GnRH signaling pathway & Endocrine system & $2.85 \mathrm{E}-02$ \\
hsa04972 & Pancreatic secretion & Digestive system & $3.08 \mathrm{E}-02$ \\
hsa05100 & Bacterial invasion of epithelial cells & Infectious diseases & $3.17 \mathrm{E}-02$ \\
hsa04730 & Long-term depression & Nervous system & $3.87 \mathrm{E}-02$ \\
\hline
\end{tabular}

with differentially expressed genes. The correlation between renal failure and cancer related biological processes may due to the dysfunction of cell cycle and DNA repair process in patients. Previous studies have demonstrated the enhanced expression of DNA repair-related proteins and induced cell cycle arrest at G1/S and G2/M in renal failure rats [20-22]. Overrepresentation of dysregulated genes in the chronic myeloid leukemia (hsa05220) pathway revealed the similar gene expression of these two diseases which may explain the causative effect of lymphocytic leukemia on renal failure [19]. These identified biological processes revealed the molecular signatures of renal failure.

To detect hub molecules, we constructed a network with proteins encoded by identified differentially expressed genes (Figure 2). Several hub molecules have been identified to play important roles in the progression of renal failure before. Take RELA for example, protein encoded by this gene is NF-kappaB p65. In consistent with our results, detection of NF-kappaB p65 based on immunohistochemical staining and ELISA suggested that NF-kappaB p65 in rat glomeruli of multiple organ failure was significantly higher than that of control group [23]. Attenuation of NF-kappaB p65 activation is effective in reducing endotoxic kidney injury [24]. Inhibition of inflammation through $\mathrm{NF}-\kappa \mathrm{B}$ also reduced renal dysfunction caused by sepsis in mice [25]. The involvement of NF-kappaB p65 in renal

Table 2 GO items enriched with differentially expressed gene

\begin{tabular}{llll}
\hline \#GO id & GO description & GO class & FDR \\
\hline GO:0005515 & protein binding & Function & $2.92 \mathrm{E}-06$ \\
GO:0005730 & nucleolus & Component & $4.54 \mathrm{E}-05$ \\
GO:0005634 & nucleus & Component & $6.16 \mathrm{E}-05$ \\
GO:0006351 & transcription, DNA-dependent & Process & $4.19 \mathrm{E}-03$ \\
GO:0002326 & B cell lineage commitment & Process & $3.72 \mathrm{E}-02$ \\
\hline
\end{tabular}

failure may be due to its interaction with inflammatory chemokines [26], such as CXCL16, which was increased in active nephrotic syndrome patients and correlated with blood lipids, urine protein and inflammation responses [27]. Genes involved in regulation of cell cycle, TP53 and $C D K 2$, were also identified as hub genes. Their involvements in renal failure through regulation of G1 cell cycle arrest were reported before [28]. Moreover, paricalcitol could prevent cisplatin-induced renal injury by suppressing the up regulation of TP53 and CDK2 [29]. Therefore, our study confirmed that these three genes may serve as potential targets for renal failure treatments. For the rest four hub genes, SRSF1, CAND1, SMURF1, and YWHAE, no previous report of their association with renal failure has been proposed before. Protein encoded by $S R S F 1$ is a member of the arginine/serine-rich splicing factor protein family. Up regulation of SRSF1 could increases the cellular pool of active p53 [30], suggesting the implication of SRSF1 in renal failure through its regulation of the p53. For SMURF1, protein encoded by this gene is an ubiquitin ligase that is specific for receptor-regulated SMAD proteins. It is reported that reduction of Smad7 due to the overexpression of Smurf1 in unilateral ureteral obstruction kidneys plays an important role in the progression of tubulointerstitial fibrosis [31], which a harmful process leading inevitably to renal function deterioration. Consistently, our analysis detected the up regulation of SMURF1, suggesting it may contribute to the progression of renal failure through its ubiquitination of SMAD7. Protein encoded by YWHAE belongs to the 14-3-3 family of proteins which mediate signal transduction by binding to phosphoserine-containing proteins. Quantitative protein expression profiling revealed that overexpression of YWHAE prompt the proliferation of renal cancer cells [32]. CAND1 may also promote the progression of renal cell carcinoma through its interaction with carbonic anhydrase IX [33]. Whether the up regulation contributes 


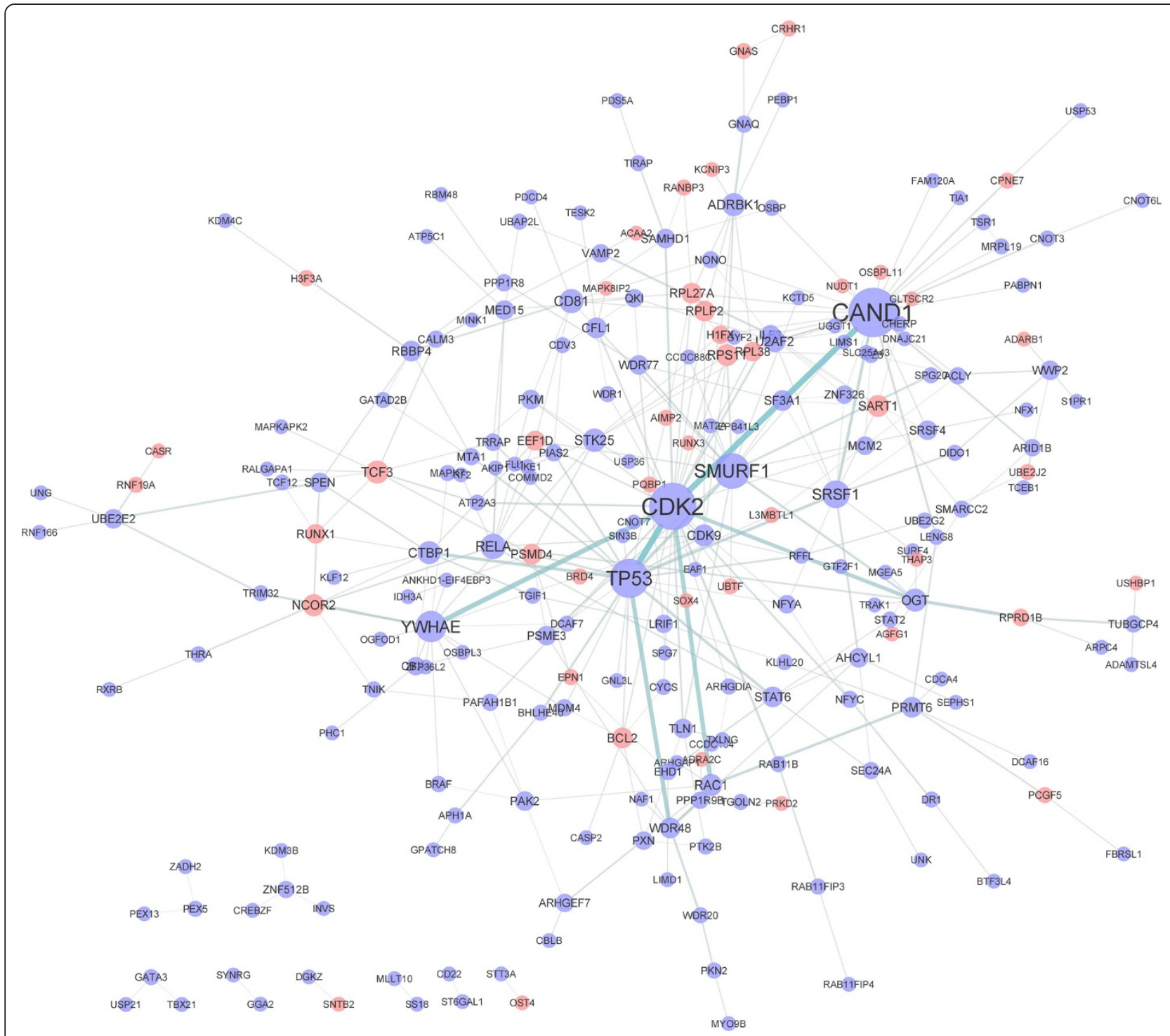

Figure 2 Interaction network constructed by proteins encoded by differentially expressed genes. Proteins with more interactions are shown in bigger size. Proteins in red are encoded by downregulated genes in patients while those in blue are encoded by upregulated genes in patients.

to the pathogenesis of renal failure needs further investigation.

\section{Conclusions}

In summary, with gene expression profile downloaded from the GEO database, we carried out PLS based analysis to identify differentially expressed genes in end-stage renal failure patients and healthy controls. Pathway and GO enrichment analyses were also implemented to capture biological relevant characters. A network of proteins encoded by differentially expressed genes was constructed to identify key molecules. Our results facilitate the disclosure of the molecular mechanism underlying renal failure progression.

\section{Consent}

Written informed consent was obtained from the patients for the publication of this report and any accompanying images.

\section{Competing interest}

The authors declare that they have no competing interests.

\section{Authors' contributions}

PM designed the research and revised the manuscript. SD drafted the manuscript. SD, YX and TH carried out data analysis. All authors read and approved the final manuscript.

Received: 17 April 2014 Accepted: 25 June 2014

Published: 5 July 2014 


\section{Reference}

1. Gross P, Schirutschke H, Barnett K: Should we prescribe blood pressure lowering drugs to every patient with advanced chronic kidney disease? A comment on two recent meta-analyses. Pol Arch Med Wewn 2009, 119:644-647.

2. Remuzzi G, Benigni A, Finkelstein FO, Grunfeld JP, Joly D, Katz I, Liu ZH, Miyata T, Perico N, Rodriguez-lturbe B, Antiga L, Schaefer F, Schieppati A, Schrier RW, Tonelli M: Kidney failure: aims for the next 10 years and barriers to success. Lancet 2013, 382:353-362.

3. Lameire NH, Bagga A, Cruz D, De Maeseneer J, Endre Z, Kellum JA, Liu KD, Mehta RL, Pannu N, Van Biesen W, Vanholder R: Acute kidney injury: an increasing global concern. Lancet 2013, 382:170-179.

4. Guebre-Egziabher F, Debard C, Drai J, Denis L, Pesenti S, Bienvenu J, Vidal H, Laville M, Fouque D: Differential dose effect of fish oil on inflammation and adipose tissue gene expression in chronic kidney disease patients. Nutrition 2013, 29:730-736.

5. Zaza G, Granata S, Rascio F, Pontrelli P, Dell'Oglio MP, Cox SN, Pertosa G, Grandaliano G, Lupo A: A specific immune transcriptomic profile discriminates chronic kidney disease patients in predialysis from hemodialyzed patients. BMC Med Genet 2013, 6:17.

6. Sun Y, Ding W, Wei Q, Shen Z, Wang C: Dysregulated gene expression of extracellular matrix and adhesion molecules in saphenous vein conduits of hemodialysis patients. J Thorac Cardiovasc Surg 2012, 144:684-689.

7. Chakraborty S, Datta S, Datta S: Surrogate variable analysis using partial least squares (SVA-PLS) in gene expression studies. Bioinformatics 2012, 28:799-806

8. Ji G, Yang Z, You W: PLS-based gene selection and identification of tumor-specific genes. leee Trans Syst Man Cybern-Part C: Appl Rev 2011, 41:830-841.

9. Gao QG, Li ZM, Wu KQ: Partial least squares based analysis of pathways in recurrent breast cancer. Eur Rev Med Pharmacol Sci 2013, 17:2159-2165.

10. Irizarry RA, Hobbs B, Collin F, Beazer-Barclay YD, Antonellis KJ, Scherf U, Speed TP: Exploration, normalization, and summaries of high density oligonucleotide array probe level data. Biostatistics 2003, 4:249-264.

11. Barker M, Rayens W: Partial least squares for discrimination. J Chemometr 2003, 17:166-173.

12. Martins JPA, Teofilo RF, Ferreira MMC: Computational performance and cross-validation error precision of five PLS algorithms using designed and real data sets. J Chemometr 2010, 24:320-332

13. Gosselin R, Rodrigue D, Duchesne C: A Bootstrap-VIP approach for selecting wavelength intervals in spectral imaging applications. Chemometr Intell Lab Syst 2010, 100:12-21

14. Kanehisa M, Goto S: KEGG: kyoto encyclopedia of genes and genomes. Nucleic Acids Res 2000, 28:27-30.

15. Ashburner M, Ball CA, Blake JA, Botstein D, Butler $H$, Cherry JM, Davis AP, Dolinski K, Dwight SS, Eppig JT, Harris MA, Hill DP, Issel-Tarver L, Kasarskis A, Lewis S, Matese JC, Richardson JE, Ringwald M, Rubin GM, Sherlock G: Gene ontology: tool for the unification of biology. The Gene Ontology Consortium. Nat Gen 2000, 25:25-29.

16. Stelzl U, Worm U, Lalowski M, Haenig C, Brembeck FH, Goehler H, Stroedicke M, Zenkner M, Schoenherr A, Koeppen S, Timm J, Mintzlaff S, Abraham C, Bock N, Kietzmann S, Goedde A, Toksöz E, Droege A, Krobitsch S, Korn B, Birchmeier W, Lehrach H, Wanker EE: A human protein-protein interaction network: a resource for annotating the proteome. Cell 2005, 122:957-968.

17. Shannon P, Markiel A, Ozier O, Baliga NS, Wang JT, Ramage D, Amin N, Schwikowski B, Ideker T: Cytoscape: a software environment for integrated models of biomolecular interaction networks. Genome Res 2003, 13:2498-2504

18. Ferreira RD, Custodio FB, Guimaraes CS, Correa RR, Reis MA: Collagenofibrotic glomerulopathy: three case reports in Brazil. Diagn Pathol 2009, 4:33.

19. Dou X, Hu H, Ju Y, Liu Y, Kang K, Zhou S, Chen W: Concurrent nephrotic syndrome and acute renal failure caused by chronic lymphocytic leukemia (CLL): a case report and literature review. Diagn Pathol 2011, 6:99.

20. Zhou H, Kato A, Yasuda H, Miyaji T, Fujigaki Y, Yamamoto T, Yonemura K, Hishida A: The induction of cell cycle regulatory and DNA repair proteins in cisplatin-induced acute renal failure. Toxicol Appl Pharmacol 2004, 200:111-120.

21. Price PM, Megyesi J, Saf Irstein RL: Cell cycle regulation: repair and regeneration in acute renal failure. Kidney Int 2004, 66:509-514.
22. Nishihara K, Masuda S, Nakagawa S, Yonezawa A, Ichimura T, Bonventre JV, Inui K: Impact of Cyclin B2 and Cell division cycle 2 on tubular hyperplasia in progressive chronic renal failure rats. Am J Physiol Renal Physiol 2010, 298:F923-F934

23. Chen XM, Du XG: [Relationship between glomerular lesion and NF-kappaB p65 activity in rat multiple organ failure caused by zymosan]. Xi Bao Yu Fen Zi Mian Yi Xue Za Zhi 2005, 21:486-488. 492.

24. Meyer-Schwesinger C, Dehde S, von Ruffer C, Gatzemeier S, Klug P, Wenzel UO, Stahl RA, Thaiss F, Meyer TN: Rho kinase inhibition attenuates LPS-induced renal failure in mice in part by attenuation of NF-kappaB p65 signaling. Am J Physiol Renal Physiol 2009, 296:F1088-F1099.

25. Coldewey SM, Rogazzo M, Collino M, Patel NS, Thiemermann C: Inhibition of IkappaB kinase reduces the multiple organ dysfunction caused by sepsis in the mouse. Dis Model Mech 2013, 6:1031-1042.

26. Lotzer K, Dopping S, Connert S, Grabner R, Spanbroek R, Lemser B, Beer M, Hildner M, Hehlgans T, van der Wall M, Mebius RE, Lovas A, Randolph GJ, Weih F, Habenicht AJ: Mouse aorta smooth muscle cells differentiate into lymphoid tissue organizer-like cells on combined tumor necrosis factor receptor-1/lymphotoxin beta-receptor NF-kappaB signaling.

Arterioscler Thromb Vasc Biol 2010, 30:395-402.

27. Zhen J, Li Q, Zhu Y, Yao X, Wang L, Zhou A, Sun S: Increased serum CXCL16 is highly correlated with blood lipids, urine protein and immune reaction in children with active nephrotic syndrome. Diagn Pathol 2014, 9:23.

28. Yang QH, Liu DW, Long Y, Liu HZ, Chai WZ, Wang XT: Acute renal failure during sepsis: potential role of cell cycle regulation. J Infect 2009, 58:459-464

29. Park JW, Cho JW, Joo SY, Kim CS, Choi JS, Bae EH, Ma SK, Kim SH, Lee J, Kim SW: Paricalcitol prevents cisplatin-induced renal injury by suppressing apoptosis and proliferation. Eur J Pharmacol 2012, 683:301-309.

30. Fregoso Ol, Das S, Akerman M, Krainer AR: Splicing-factor oncoprotein SRSF1 stabilizes p53 via RPL5 and induces cellular senescence. Mol Cell 2013, 50:56-66.

31. Fukasawa H, Yamamoto T, Togawa A, Ohashi N, Fujigaki Y, Oda T, Uchida C, Kitagawa K, Hattori T, Suzuki S, Kitagawa M, Hishida A: Down-regulation of Smad7 expression by ubiquitin-dependent degradation contributes to renal fibrosis in obstructive nephropathy in mice. Proc Natl Acad Sci U S A 2004, 101:8687-8692

32. Liang S, Xu Y, Shen G, Liu Q, Zhao X, Xu Z, Xie X, Gong F, Li R, Wei Y: Quantitative protein expression profiling of 14-3-3 isoforms in human renal carcinoma shows 14-3-3 epsilon is involved in limitedly increasing renal cell proliferation. Electrophoresis 2009, 30:4152-4162.

33. Buanne P, Renzone G, Monteleone F, Vitale M, Monti SM, Sandomenico A, Garbi C, Montanaro D, Accardo M, Troncone G, Zatovicova M, Csaderova L, Supuran CT, Pastorekova S, Scaloni A, De Simone G, Zambrano N: Characterization of carbonic anhydrase IX interactome reveals proteins assisting its nuclear localization in hypoxic cells. J Proteome Res 2013, 12:282-292

doi:10.1186/1746-1596-9-137

Cite this article as: Ding et al:: Partial least squares based gene expression analysis in renal failure. Diagnostic Pathology 2014 9:137.

\section{Submit your next manuscript to BioMed Central and take full advantage of:}

- Convenient online submission

- Thorough peer review

- No space constraints or color figure charges

- Immediate publication on acceptance

- Inclusion in PubMed, CAS, Scopus and Google Scholar

- Research which is freely available for redistribution 